\title{
Prevention and recovery for peritoneal injury during totally extraperitoneal inguinal hernia repair: A single-center retrospective cohort study
}

Ryohei Nishiguchi ( $\nabla$ ryohei.nishiguchi@twmu.ac.jp )

Department of Surgery, Tokyo Women's Medical University Medical Center East

\section{Shigehiro Kojima}

Department of Surgery, Sainokuni Higashiomiya Medical Center

\section{Shinichi Asaka}

Department of Surgery, Tokyo Women's Medical University Medical Center East

\section{Hiroyuki Maeda}

Department of Surgery, Tokyo Women's Medical University Medical Center East

\section{Takebumi Usui}

Department of Surgery, Tokyo Women's Medical University Medical Center East

\section{Hajime Yokomizo}

Department of Surgery, Tokyo Women's Medical University Medical Center East

\section{Takeshi Shimakawa}

Department of Surgery, Tokyo Women's Medical University Medical Center East

\section{Takao Katsube}

Department of Surgery, Tokyo Women's Medical University Medical Center East

\section{Shunichi Shiozawa}

Department of Surgery, Tokyo Women's Medical University Medical Center East

\section{Research Article}

Keywords: Inguinal hernia, lateral thermal spread, peritoneal injury, thermal injury, totally extraperitoneal (TEP) repair, ultrasonically activated device (USAD)

Posted Date: December 15th, 2020

DOI: https://doi.org/10.21203/rs.3.rs-121769/v1

License: (c) (1) This work is licensed under a Creative Commons Attribution 4.0 International License. Read Full License 


\section{Abstract}

Background During totally extraperitoneal (TEP) repair, peritoneal injury (PI) may result in technical difficulty due to the imparied working space inside the preperitoneal space. We aimed to clarify the factors causing Pls by focusing on the size of the hernia and to provide the prevention and recovery method for PI.

Methods A total of 71 inguinal hernia with unilateral TEP repairs were classified by the size of hernia; Small group $(<1 \mathrm{~cm}, \mathrm{n}=16)$ and Large group $(\geqq 1 \mathrm{~cm}, \mathrm{n}=55)$. Patient characteristics and surgical outcomes were analyzed retrospectively. TEP procedure was broke down into five phases (e.g. Phase 3 was a period from starting the dissection to transection of hernia sac) in the PI cases of the Large group $(n=21)$. Common site of PI, maneuver and recovery method were further analyzed.

Results Operative time $(P<0.0001)$ and $\mathrm{PI}$ rate $(P=0.015)$ were higher in the Large group. PI cases in the Large group showed a higher PI rate in Phase $3(P=0.036)$ and PI mostly occurred by a sharp dissection of the medial side of hernia sac with using an ultrasonically activated device. In terms of recovery methods, pre-tied suture loop ligation and endoscopic suturing tended to be faster than doing nothing and conversion to TAPP but showed no significance $(P=0.059)$.

Conclusions Hernia size and a sharp dissection during the medial side of the hernia sac may be important factors causing Pls. A careful dissection combined with an appropriate blunt dissection is required for the prevention of $\mathrm{PI}$ because of the strong adhesion at the medial side and thin peritoneum in most cases of large hernia sacs. In cases of Pl, extension of operative time could be prevented by ligation or suturing of the peritoneal defect.

\section{Background}

Totally extraperitoneal (TEP) repair is one of the first recommendation of adult inguinal hernia repairs along with transabdominal preperitoneal (TAPP) repair and Lichtenstein repair, followed by the European Hernia Society (EHS) guidelines [1, 2]. However, the percentage of TEP repair performed over the decade in Japan was $5.9 \%$ (2007) to $8.2 \%$ (2017) which was far less than TAPP repair, which increased explosively from $4.5 \%$ (2007) to $37.7 \%$ (2017) [3]. One of the reasons for this fact may be due to the long and steep learning curve and narrow and limited working space inside the preperitoneal space for TEP repairs $[4,5]$, which is an unfamiliar technique for surgeons who are usually familiar to the intraperitoneal view.

Peritoneal injury (PI) is a major intraoperative complication specific to TEP repair which leads to a loss of the preperitoneal space due to pneumoperitoneum. Since TEP repair is a technique with a limited working space inside the preperitoneal space, $\mathrm{PI}$ is a critical factor making the rest of the surgical process difficult. Our aim of this study was to clarify the factors causing Pls by focusing on the size of hernia and to identify the prevention and recovery method for PI upon TEP repairs. 


\section{Methods}

[Patient]

A total of 78 adult inguinal hernia patients underwent TEP repair at Tokyo Women's Medical University Medical Center East between June 2017 and April 2020. One patient without a preoperative computed tomography (CT) scan and six patients who had bilateral hernia were excluded from the study. Therefore, 71 patients (64 men, 7 women) were enrolled into the study (Figure 1). Patients were classified into two groups by the size of hernia as followed. 1) Small group $(n=16)$ with a hernia smaller than $1 \mathrm{~cm}$ and 2) Large group $(n=55)$ with a hernia $1 \mathrm{~cm}$ or larger. Patient characteristics and surgical outcomes (operative time, blood loss and perioperative complications) were analyzed between the two groups. We further analyzed the PI cases in the Large group $(\mathrm{n}=21)$ by breaking down the TEP procedure into five surgical phases described below, and focused on the common site of $\mathrm{Pl}$, maneuver and recovery method during each phase. Patient selection for TEP repairs were carried out based on the European Hernia Society (EHS) guidelines [1, 2]. The size of hernia was defined based on the Japan Hernia Society (JHS) Classification for groin hernia [6].

[Surgeon backgrounds]

Four surgeons who had not experienced TEP repair more than 15 cases, but had a satisfactory experience with other gastrointestinal and laparoscopic surgeries were assigned to the study. Each surgeon performed 15 to 25 TEP repairs in this study. Prior to the study, three surgeons were trained under expert surgeons who had performed more than 1,000 cases of TEP repair in another institution.

[Operative Techniques]

A 12-mm blunt-tip trocar was inserted into the preperitoneal space via a longitudinal incision on the lower aspect of the umbilicus. We broke down the TEP procedure into five phases described as followed. Phase 1: A 5-mm working port was inserted directly below the first trocar. After dissecting the preperitoneal space toward the Cooper's ligament, an additional 5-mm working port was inserted three finger widths above the pubic symphysis and the inferior epigastric vessels were confirmed. Phase 2: The lateral side of the preperitoneal space was dissected until the anterior superior iliac spine and the peritoneal edge was defined as a landmark. For mesh placement, the lateral edge of the arcuate line was partially incised. Phase 3: The indirect hernia sac was dissected followed by ligation and transection of the sac. Phase 4: Parietalization of the testicular vessels and the spermatic cord (round ligament for female patients) was performed by separating the dorsal side of the preperitoneal space. Phase 5: A Parietex ${ }^{\mathrm{TM}}$ Anatomical Mesh (Medtronic, Dublin, Ireland) was placed on the myopectineal orfice. The mesh was fixed on the Cooper's ligament and medial / lateral side of the inferior epigastric vessels using an AbsorbaTack ${ }^{\mathrm{TM}}$ (Medtronic). The ultrasonically activated device (USAD) mainly used in this study was SONICBEATTM (OLYMPUS, Tokyo, Japan) with an ultrasonic generator USG-400 (OLYMPUS). Maneuver during PI was devided into a blunt dissection and a sharp dissection. A blunt dissection was mainly performed utilizing a Kelly forceps and a grasping forceps. A sharp dissection was mainly performed utilizing an USAD or a 
monopolar scissors. Recovery methods for the PI were carried out by a pre-tied suture loop ligation with ENDOLOOP ${ }^{\mathrm{TM}}$ (Johnson \& Johnson, New Brunswick, NJ) or an endoscopic continuous suturing with 4-0 PDS $^{\mathrm{TM}}$ (Johnson \& Johnson). In cases with serious peritoneal injuries or adhesions in which TEP repair was hard to continue, the procedure was converted to TAPP repair.

[Statistical Analysis]

Continuous data are presented as median or mean \pm SD. Continuous variables were nonparametrically analyzed using Mann-Whitney $U$ test or Kruskal-Wallis test. Categorical variables were compared using chi square test and probabilities were evaluated by Pearson's chi square test. Correlations between the categorical variables were assessed using Pearson correlation coefficient. A $P$ value less than 0.05 was considered significant. All statistical data were generated using JMP Pro 15 (SAS Institute, Cary, NC).

\section{Results}

[Patient characteristics]

Preoperative patient characteristics between the Small group and Large group are presented in Table 1. There was no significant difference in age, gender, body mass index (BMI) and comorbidity between the groups. In terms of the hernia characteristics, there was no significant difference in the location of hernia. However, there was a significant difference in the type of hernia (Nyhus classification, $P<0.0001$ ).

[Surgical outcomes]

Operative time was significantly longer ( $86.5 \mathrm{~min}$ vs $129 \mathrm{~min}, P<0.0001)$, PI rate was significantly higher (6.25\% vs $38.2 \%, P=0.015)$, and blood loss tended to increase but no significance $(1.8 \pm 1.7 \mathrm{~mL}$ vs $2.3 \pm 1.4 \mathrm{~mL}, P=0.054)$ in the Large group. There was no significant difference in the other perioperative complications including conversion to TAPP, bleeding, seroma, wound infection, chronic pain and postoperative recurrence during the 30 days follow-up period (Table 2).

In terms of the PI location during each surgical phase in the Large group $(n=21)$, we clarified that the PI rate was significantly higher in Phase $3(52.4 \%, P=0.036)$ compared to the other phases (Table 3$)$. We further reviewed the PI cases in the Large group $(n=21)$ during each phase in terms of maneuver method, utilized device and recovery method (Table 4). Based on the data of Table 4, we focused on the maneuver method and injury location during Phase 3 (Table 5). We recognized that $81.8 \%$ of the maneuver during Phase 3 was a sharp dissection utilizing an ultrasonically activated device (USAD) $(P=0.035)$ and $72.7 \%$ of the injury occurred during the dissection of the medial side of the hernia sac $(P=0.132)$.

Recovery methods used for the PI included pre-tied suture loop ligation ( $n=11)$, endoscopic suturing $(n=3)$, conversion to TAPP $(n=4)$ and three cases were done nothing (Table 4). Regarding each recovery methods, comparison of the operative time of TEP repairs utilizing different recovery methods for the closure of the PI showed that pre-tied suture loop ligation (123 min (87-156)) was faster than doing 
nothing (157 min (144-181)) for small Pls and endoscopic suturing (136 min (85-166)) was faster than conversion to TAPP (186 min (117-255)) for larger Pls (Figure 2, $P=0.059$ ).

\section{Discussion}

In many studies, TEP repair is reported as a safe and effective procedure for treating inguinal hernias, and the advantages over open repair are less pain, better cosmetic outcomes and shorter recovery [7-9]. However, many institutions hesitate to introduce TEP repair because of the limited working space and the peculiarity of the anatomical landmarks $[1,10]$. Compared to TEP repair, TAPP repair has the advantage of a clear field of view inside the pelvic cavity similar to other laparoscopic surgeries. However, TAPP repair is associated with higher rates of port-site hernias and visceral injuries [11] and others report that TAPP repair has the possibility causing more adhesions, leading to intestinal obstruction[12].

$\mathrm{PI}$ is an intraoperative complication specific to TEP repair which occurs during the dissection of the preperitoneal space. Several studies have reported that the incidence of PI ranges from $10 \%$ to $65 \%$, and the rate increases in recurrent and bilateral hernias [9, 13-16]. Pneumoperitoneum caused by PI induces loss of the working space inside the preperitoneal space, leading to prolongation of operative time and difficulty in performing the operation safely. In cases with small PIs, closure of the defect is preferred [13], however, it is difficult to control large PIs and conversion to anterior approach or TAPP repair may be needed in severe cases $[9,11,15]$. In the present study, the total PI rate was $31 \%$, and the conversion rate was $5.6 \%$ which was a major cause of prolonging the operative time.

To clarify the factors causing PIs, we focused on the size of hernia and analyzed the PI cases in large hernias by breaking down the TEP procedure into five phases. By this way, we clarified that the PI was likely to occur during the dissection of the medial side of the hernia sac by a sharp dissection using an USAD. Based on these findings, we made two presumptions causing PI during the dissection of the hernia sac as followed. 1) Maneuver during the hernia sac dissection. 2) Possibility of thermal injury due to the narrow working space. The medial side of the hernia sac is relatively adherent to the underlying structures such as the vas deferens. Therefore, dissecting the adherent membrane of the hernia sac, especially a sharp dissection utilizing an USAD may be one factor causing Pls. For presumption 2), we presumed that the large hernia sac itself caused the narrowing of the working space, leading to an increase of injury. Additionally, when the hernia sac is large, the peritoneum is originally thin and weak, causing more opportunity of injury. We also considered the possibility of thermal injury to the peritoneum since the active blade of the USAD is long (15-20 mm), and the effect of lateral thermal spread to the surrounding tissue is wide $(2-5 \mathrm{~mm})$ enough to injure the peritoneum in a narrow and limited space $[17,18]$. Our future direction is to initiate a new method to quantify the working space inside the preperitoneal space and to determine whether the hernia size and other factors affects the working space.

Based on the presumptions causing PI, we next considered the prevention method for PI during the hernia sac dissection and the remaining preperitoneal space dissection. When utilizing an USAD in medium power settings (60-70\% power), the mean temperature of the active blade elevates around $80^{\circ} \mathrm{C}$ 
with an application time of 5 seconds, and reaches to a plateau around $80-100^{\circ} \mathrm{C}$ when applied for a longer period of time. However, the mean temperature can easily elevate over $100^{\circ} \mathrm{C}$ even with an application time of 5 seconds and over in maxium power settings $(100 \%)[17,18]$. Since protein coagulation and denaturation begins over $60^{\circ} \mathrm{C}$ and collagen denaturation and cell membrane permeabilization begins over $80^{\circ} \mathrm{C}$ [19], we believe that minimizing the application time (within 5 seconds) as suggested by Pogerlic et al. [20], and minimizing the output power (60-70\% power) should be useful to prevent maxium heating of the active blade. Moreover, in order to prevent thermal injury and cavitation damage to the surrounding tissue, maintaining a safety margin with a proper traction should always be considered. Furthermore, the hernia sac dissection should be carried out properly by a combination of a blunt and sharp dissection while performing a delicate hemostatic procedure. Under these methods, we believe that the peritoneum injury will be minimized, leading to the safety of the intraabdominal organs, which is a significant advantage of TEP repairs compared to TAPP repairs.

As shown in our study, recovery methods for the PI was effective regardless of the size of the defect. To control the narrow working space created by $\mathrm{PI}$, recovery methods should be strongly considered. Several studies have reported repairments of the peritoneum utilizing endoscopic metal / non-metal stapling, endoscopic suturing and pre-tied suture loop ligation $[13,14]$. We considered that the PI defect should be repaired because of the two points as followed. 1) Narrowing of the working space due to pneumoperitoneum causes decrease of maneuverability, resulting in prolongation of operative time. 2) Can be causes of postoperative internal hernia and adhesive intestinal obstruction [12]. Moreover, when the peritoneal defect is large, conversion to TAPP may cause 1) Prolongation of operative time. 2) Effect the cosmetic outcomes by adding another skin incision. 3) Possibility of adhesive intestinal obstruction. Therefore, as long as the working space is maintained, we recommend repairment of the peritoneum by continuous suturing. In cases which the working space is difficult to maintain, a continuous suture while deaerating the peritoneal cavity may be effective.

Several studies have reported that the PI rate is lower in an expert surgeon compared to trainees $[15,21]$. In the present study, Pls mostly occurred during the dissection of the medial side of the hernia sac, which a forced dissection of the adherent membrane is needed. Not only a sharp dissection utilizing an USAD, but also a forceful dissection with an unbalanced traction and countertraction may easily injure the peritoneum. We considered that the understanding of the characteristics among each energy device and the maneuver based on a proper traction was the difference between our institution and expert surgeons.

This study has some limitations including its retrospective design with small number of cases and lack of the long-term follow-up. The long-term follow-up was limited to complication cases occurring within 30 days of the procedure. Additionally, we are a developing institution in terms of TEP repair, which the learning curve may be unstable during the study.

\section{Conclusions}


We clarified that PI is more likely to occur during the dissection of the hernia sac. Even more, size of the hernia and the dissection method of the hernia sac may contribute to the PI during TEP repairs. For prevention of $\mathrm{Pl}$, a careful dissection combined with an appropriate blunt dissection is required because of the strong adhesion at the medial side and thin peritoneum in most cases of large hernia sacs. In cases of $\mathrm{Pl}$, prolongation of operative time could be prevented by ligation or suturing of the peritoneal defect.

\section{List Of Abbreviations}

TEP: Totally extraperitoneal, TAPP: Transabdominal preperitoneal, EHS: European Hernia Society, JHS: Japan Hernia Society, PI: Peritoneal injury, CT: Computed tomography, USAD: Ultrasonically activated device, BMI: Body mass index

\section{Declarations}

\section{Ethics approval, guidelines and consent to participate:}

The protocol of this study was approved by the institutional review board in Tokyo Women's Medical University (Approved No.5733). All methods were carried out based on the European Hernia Society (EHS) and Japan Hernia Society (JHS) guidelines. Informed consent was waived by the Tokyo Women's Medical University Ethics Committee. As an alternative, the opt-out consent was approved by the committee and obtained via our websites, where permission was requested for the use of the participants' personal information in this study.

\section{Consent for publication:}

Not applicable.

\section{Availability of data and meterials:}

The datasets used and analysed during the current study are available from the corresponding author on reasonable request.

\section{Competing interests:}

The authors declare that they have no competing interests.

\section{Funding:}

Not applicable.

\section{Authors' contributions:}


RN designed, collected data and drafted the manuscript. SK and SA contributed to design and draft the manuscript. RN, SA, HM and TS performed the operation. TU, HY and TK helped to draft the manuscript. SS provided final approval of the version to be published. All authors have approved the manuscript and agree with submission to the BMC Surgery.

\section{Acknowledgments:}

The authors thank Dr. Tsuguo Sakamoto as an expert to teach the TEP procedure and Dr. Masano Sagawa for managing the IRB procedure.

\section{References}

1. Simons MP, Aufenacker T, Bay-Nielsen M, Bouillot JL, Campanelli G, Conze J, de Lange D, Fortelny R, Heikkinen T, Kingsnorth A et al: European Hernia Society guidelines on the treatment of inguinal hernia in adult patients. Hernia 2009, 13(4):343-403.

2. Miserez M, Peeters E, Aufenacker T, Bouillot JL, Campanelli G, Conze J, Fortelny R, Heikkinen T, Jorgensen LN, Kukleta $J$ et al: Update with level 1 studies of the European Hernia Society guidelines on the treatment of inguinal hernia in adult patients. Hernia 2014, 18(2):151-163.

3. Inomata M, Shiroshita H, Uchida H, Bandoh T, Akira S, Yamaguchi S, Kurokawa Y, Seki Y, Eguchi S, Wada $\mathrm{N}$ et al: Current status of endoscopic surgery in Japan: The 14th National Survey of Endoscopic Surgery by the Japan Society for Endoscopic Surgery. Asian J Endosc Surg 2020, 13(1):7-18.

4. Putnis S, Berney CR: Totally extraperitoneal repair of inguinal hernia: techniques and pitfalls of a challenging procedure. Langenbecks Arch Surg 2012, 397(8):1343-1351.

5. Suguita FY, Essu FF, Oliveira LT, luamoto LR, Kato JM, Torsani MB, Franco AS, Meyer A, Andraus W: Learning curve takes 65 repetitions of totally extraperitoneal laparoscopy on inguinal hernias for reduction of operating time and complications. Surg Endosc 2017, 31(10):3939-3945.

6. Japanese hernia society: Japanese hernia society classification of inguinal hernia.In.;2009. http://jhs.mas-sys.com/classification.html Accessed 8 Dec 2020.

7. Chung RS, Rowland DY: Meta-analyses of randomized controlled trials of laparoscopic vs conventional inguinal hernia repairs. Surg Endosc 1999, 13(7):689-694.

8. Heikkinen TJ, Haukipuro K, Hulkko A: A cost and outcome comparison between laparoscopic and Lichtenstein hernia operations in a day-case unit. A randomized prospective study. Surg EndosC 1998, 12(10):1199-1203.

9. Liem MS, van der Graaf Y, van Steensel CJ, Boelhouwer RU, Clevers GJ, Meijer WS, Stassen LP, Vente JP, Weidema WF, Schrijvers AJ et al: Comparison of conventional anterior surgery and laparoscopic surgery for inguinal-hernia repair. N Engl J Med 1997, 336(22):1541-1547.

10. Lau H, Patil NG, Yuen WK, Lee F: Learning curve for unilateral endoscopic totally extraperitoneal (TEP) inguinal hernioplasty. Surg Endosc 2002, 16(12):1724-1728. 
11. Schmedt CG, Sauerland S, Bittner R: Comparison of endoscopic procedures vs Lichtenstein and other open mesh techniques for inguinal hernia repair: a meta-analysis of randomized controlled trials. Surg Endosc 2005, 19(2):188-199.

12. McCormack K, Wake BL, Fraser C, Vale L, Perez J, Grant A: Transabdominal pre-peritoneal (TAPP) versus totally extraperitoneal (TEP) laparoscopic techniques for inguinal hernia repair: a systematic review. Hernia 2005, 9(2):109-114.

13. Lau H, Patil NG, Yuen WK, Lee F: Management of peritoneal tear during endoscopic extraperitoneal inguinal hernioplasty. Surg Endosc 2002, 16(10):1474-1477.

14. Nitta T, Kataoka J, Ohta M, Fujii K, Takashima Y, Ishibashi T: Usefulness of repair using Hem-o-lok for peritoneal tear as a complication of totally extraperitoneal repair: Case series. Ann Med Surg (Lond) 2020, 49:5-8.

15. Muzio G, Bernard K, Polliand C, Rizk N, Champault G: Impact of peritoneal tears on the outcome and late results (4 years) of endoscopic totally extra-peritoneal inguinal hernioplasty. Hernia 2006, 10(5):426-429.

16. Knook MT, Weidema WF, Stassen LP, van Steensel CJ: Endoscopic total extraperitoneal repair of primary and recurrent inguinal hernias. Surg Endosc 1999, 13(5):507-511.

17. Adamczewski Z, Krol A, Kaluzna-Markowska K, Brzezinski J, Lewinski A, Dedecjus M: Lateral spread of heat during thyroidectomy using different haemostatic devices. Ann Agric Environ Med 2015, 22(3):491-494.

18. Kinoshita T, Kanehira E, Omura K, Kawakami K, Watanabe Y: Experimental study on heat production by a 23.5-kHz ultrasonically activated device for endoscopic surgery. Surg Endosc 1999, 13(6):621625.

19. Reidenbach H-D, Buess G: Ancillary technology: electrocautery, thermocoagulation and laser. In: Operative manual of endoscopic surgery. edn.: Springer; 1992: 44-60.

20. Pogorelic Z, Perko Z, Druzijanic N, Tomic S, Mrklic I: How to prevent lateral thermal damage to tissue using the harmonic scalpel: experimental study on pig small intestine and abdominal wall. Eur Surg Res 2009, 43(2):235-240.

21. Barrat C, Voreux JP, Occelli G, Catheline JM, Champault G: [Effects of surgical education and training on the results of laparoscopic treatment of inguinal hernias]. Chirurgie 1999, 124(3):298-303.

\section{Tables}

Tables 1-5 are available in the Supplementary Files.

\section{Figures}




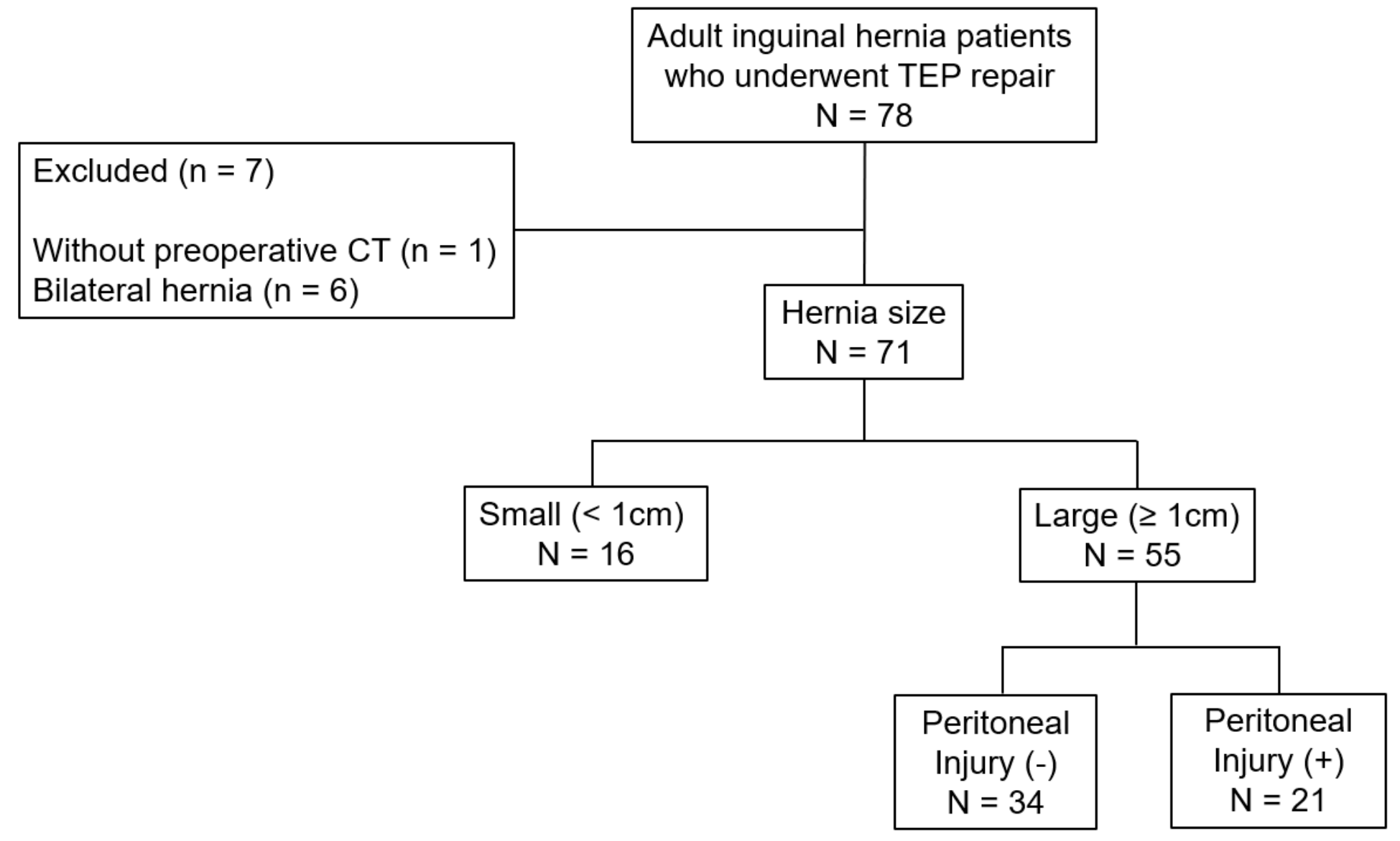

\section{Figure 1}

Flowchart of patient selection. There were 78 patients who underwent totally extraperitoneal repair. 7 patients were excluded from the study. The remaining patients were classified into two groups depending on the hernia size (Small, Large). Finally, patients in the Large group were classified into group without peritoneal injury $(n=34)$ and with peritoneal injury $(n=21)$. 


$$
P=0.059
$$

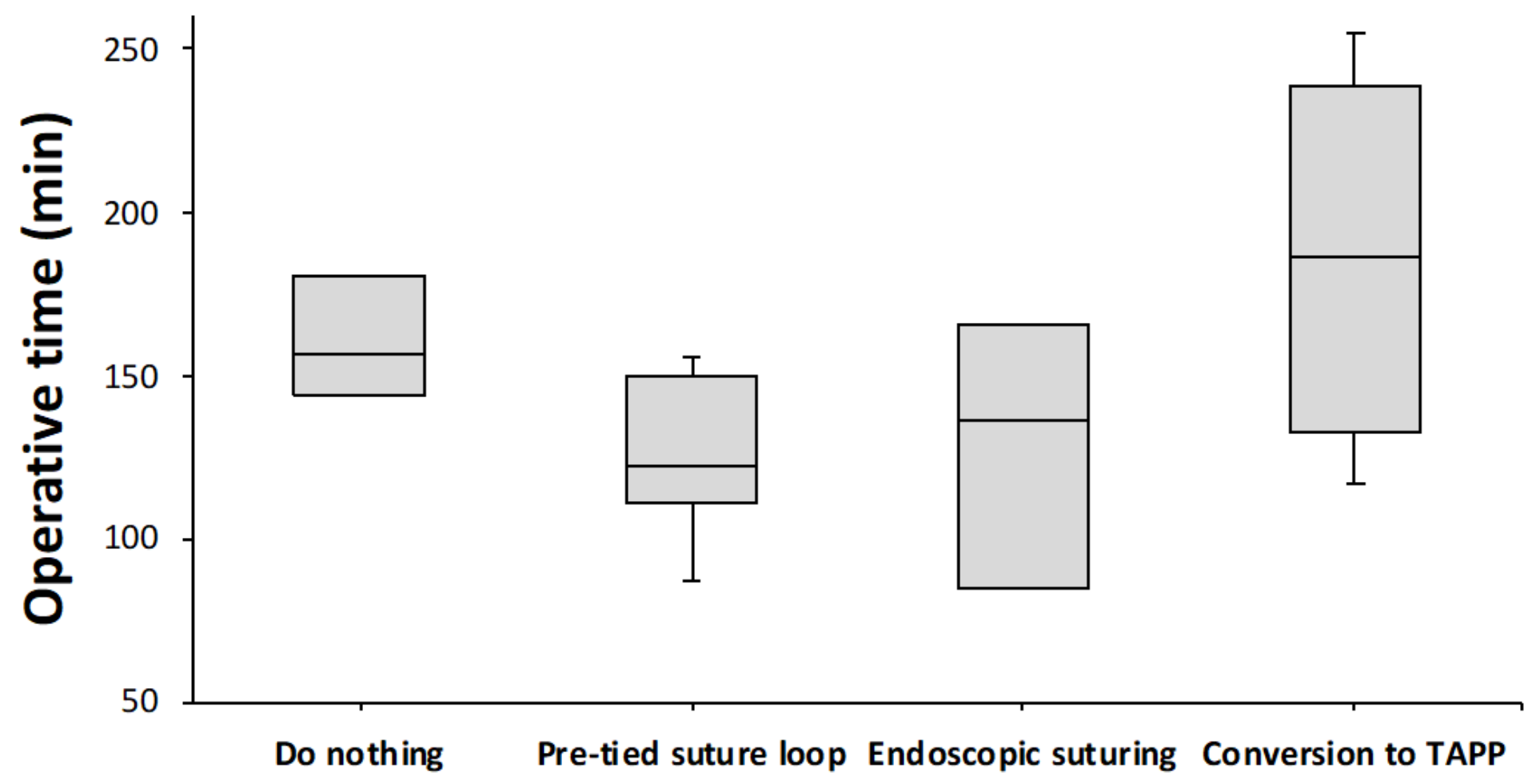

Figure 2

Comparison of operative time for recovery methods of the peritoneal injury. Operative time of totally extraperitoneal repairs utilizing different recovery methods for the closure of peritoneal injury was compared. The recovery methods included doing nothing, pre-tied suture loop ligation, endoscopic suturing and conversion to transabdominal preperitoneal repair.

\section{Supplementary Files}

This is a list of supplementary files associated with this preprint. Click to download.

- Table.xlsx 